\title{
UNIVERSITYOF
}

FORWARD

THINKING

WESTMINSTER用

WestminsterResearch

http://www.westminster.ac.uk/westminsterresearch

\section{Application of Topological Operators over Data from InterCriteria} Analysis

Roeva, O., Vassilev, P. and Chountas, P.

This is an author's accepted manuscript of an article published in Christiansen $\mathrm{H}$., Jaudoin H., Chountas P., Andreasen T., Legind Larsen H. (eds) Flexible Query Answering Systems. FQAS 2017. Lecture Notes in Computer Science, vol 10333. Springer, Cham.

The final publication is available at Springer via:

https://dx.doi.org/10.1007/978-3-319-59692-1 19

The WestminsterResearch online digital archive at the University of Westminster aims to make the research output of the University available to a wider audience. Copyright and Moral Rights remain with the authors and/or copyright owners.

Whilst further distribution of specific materials from within this archive is forbidden, you may freely distribute the URL of WestminsterResearch: ((http://westminsterresearch.wmin.ac.uk/)).

In case of abuse or copyright appearing without permission e-mail repository@westminster.ac.uk 


\title{
Generalized Net Model of Muscle Pain Diagnosing
}

\author{
Simeon Ribagin ${ }^{1}$, Panagiotis Chountas $^{2}$, Tania Pencheva ${ }^{1}$ \\ ${ }^{1}$ Institute of Biophysics and Biomedical Engineering, \\ Bulgarian Academy of Sciences, Sofia, Bulgaria \\ sim_ribagin@mail.bg, tania.pencheva@biomed.bas.bg \\ ${ }^{2}$ University of Westminster, Faculty of Science and Technology, \\ FST, Dept. of Computer Science, London, UK \\ P.I.Chountas@westminster.ac.uk
}

\begin{abstract}
Pain is the most common symptom of the many musculoskeletal pathologies. Musculoskeletal pain affects the muscles, ligaments, tendons, nerves and bones and might be caused by diverse factors. Musculoskeletal pain ranges from mild to severe. It can be local or diffuse, and acute or chronic. Due to the wide range of conditions that may cause such a symptom, diagnosing process is challenging and a systematic approach is necessary. In this investigation we present a successful example of generalized nets application in medical diagnosing and propose a novel approach leading to the appropriate diagnostic considerations. The method proposed in this investigation accurately identifies the various steps during the muscle pain diagnosing process and significantly improves the health care level. Obtained so far results could be used to assist in the decision making in the diagnostic processes.
\end{abstract}

Keywords: Musculoskeletel Pain, Generalized Nets

\section{Introduction}

The diagnosis of musculoskeletal pain is made on the basis of the medical history, clinical examinations, diagnostic imaging techniques and simple laboratory investigations. The basic issue associated with muscle pain diagnosing is to find the early pointers to a likely diagnosis. Then as part of the assessment, it is essential to establish a logical step-by-step approach to the medical history as well as a series of screening investigations. Muscle pain may arise due to injury or overexertion, infections, inflammatory or systemic conditions. A number of conditions can be associated with muscle pain, such as viral infections, fibromyalgia, autoimmune disorders, drug-induced disorders, metabolic disorders, etc. The purpose of the present study is to give an example how the apparatus of generalized nets might be successfully applied to medical diagnosing and as such to be proposed as a novel mathematical approach for diagnosing the causes of the muscle pain. Generalized nets (GNs) (see [1], [2]) are an apparatus for modeling of parallel and concurrent processes, developed as an extension of the concept of Petri nets 
and some of their modifications. In general, the GNs may or may not have some of the components in their definition. GNs without some of their components form special classes called reduced GNs [2]. The presented GN-model shows similar features with previous models for medical diagnosing [3-5] but this is the first one highlighting the diagnostic algorithm for patients with muscle pain.

\section{Generalized Net Model of Muscle Pain Diagnosing}

A reduced GN-model which represents the plan for muscle pain diagnosing is developed here. The proposed GN-model is shown in Fig. 1.

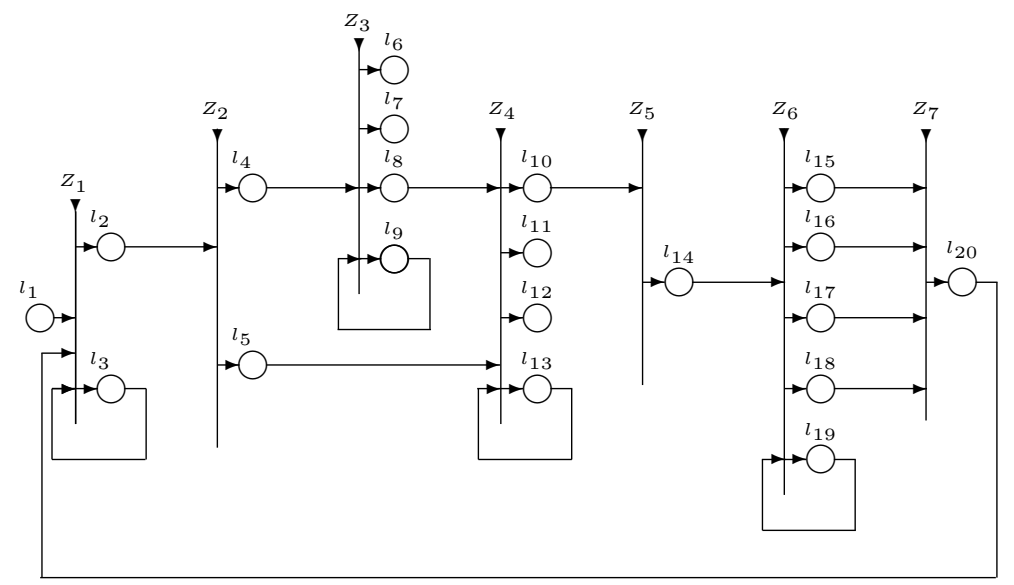

Figure 1. GN-model of muscle pain diagnosing

The GN-model has 7 transitions and 20 places with the following meanings:

- $Z_{1}$ represents the personal data of the patient;

- $Z_{2}$ - the current localization of the symptoms;

- $Z_{3}$ - the results from the medical history;

- $Z_{4}$ - the results from the physical examination;

- $Z_{5}$ - the set of laboratory tests;

- $Z_{6}$ - the results from the laboratory tests and possible diagnosis;

- $Z_{7}$ - the final diagnosis.

The GN-model contains 5 types of tokens: $\alpha, \beta, \mu, \nu$ and $\pi$. Some of the model transitions contain the so called "special place" where a token stays and collects information about the specific parts of the diagnosing process which are represented as follows: 
- place $l_{3}$ collects the overall information obtained from the diagnostics steps in the personal record (personal data);

- place $l_{9}$ - information obtained from the history of the patient;

- place $l_{13}$ - information about the results from physical examinations;

- place $l_{19}$ - information about the results from the laboratory tests.

During the GM-model functioning, the $\alpha$-tokens will unite with the tokens from the other types: $\beta, \mu, \nu$ and $\pi$. After that, some of these tokens can split in order to generate new $\alpha$-tokens obtaining corresponding characteristics. When there are some $\alpha$-tokens $\left(\alpha_{1}, \alpha_{2}, \alpha_{3}\right.$ and, eventually, $\left.\alpha_{4}\right)$, on the next timemoment all they will unite with a token from another type.

The token $\alpha$ enters the net in place $l_{1}$ with an initial characteristic:

"patient with muscle pain".

The transition $Z_{1}$ has the following form:

$$
Z_{1}=\left\langle\left\{l_{1}, l_{3}, l_{20}\right\},\left\{l_{2}, l_{3}\right\}, r_{1}\right\rangle
$$

where

$$
r_{1}=\begin{array}{c|cc} 
& l_{2} \quad l_{3} \\
\hline l_{1} & \text { false true } \\
l_{3} & W_{3,2} \text { true } \\
l_{20} & \text { false true }
\end{array}
$$

- $W_{3,2}=$ "information about the current symptoms, medical history and physical examination is necessary".

The tokens from the three input places of transition $Z_{1}$ enter place $l_{3}$ and unite with token $\beta$ with the above mentioned characteristic. On the next timemoment, token $\beta$ splits to two tokens - the same token $\beta$ and token $\alpha_{1}$. When predicate $W_{3,2}$ is true, the token $\alpha_{1}$ enters place $l_{2}$ and there it obtains a characteristic:

"obtain information about the current symptoms, medical history and physical examination".

The transition $Z_{2}$ has the following form:

$$
Z_{2}=\left\langle\left\{l_{2}\right\},\left\{l_{4}, l_{5}\right\}, r_{2}\right\rangle
$$

where

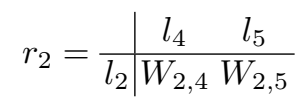

and 
- $W_{2,4}=$ "the muscle pain is local";

- $W_{2,5}=$ "the muscle pain is diffuse with no history of trauma, overuse and stress".

When the predicate $W_{2,4}$ is true, token $\alpha_{1}$ enters place $l_{4}$ and there it obtains a characteristic:

"obtain information from the medical hystory of the patient".

When the predicate $W_{2,5}$ is true, token $\alpha_{1}$ enters place $l_{5}$ and there it obtains a characteristic:

$$
\text { "perform physical examination". }
$$

The transition $Z_{3}$ has the following form:

$$
Z_{3}=\left\langle\left\{l_{4}, l_{9}\right\},\left\{l_{6}, l_{7}, l_{8}, l_{9}\right\}, r_{3}\right\rangle
$$

where

$$
r_{3}=\begin{array}{c|cccc} 
& l_{6} & l_{7} & l_{8} & l_{9} \\
\hline l_{4} & \text { false false false true } \\
l_{9} & W_{9,6} & W_{9,7} & W_{9,8} & \text { true }
\end{array}
$$

and

- $W_{9,6}=$ "there is a history of trauma";

- $W_{9,7}=$ "there is a history of recent overexertion and stress";

- $W_{9,8}=\neg W_{9,6} \vee \neg W_{9,7}$.

The tokens from all input places of transition $Z_{3}$ enter place $l_{9}$ and unite with token $\mu$ with the characteristic as mentioned above. On the next time-moment, token $\mu$ splits to two tokens - the same token $\mu$ that stays permanently in the place $l_{9}$, and token $\alpha_{1}$. When the predicate $W_{9,6}$ is true, token $\alpha_{1}$ enters place $l_{6}$ and there it obtains a characteristic:

"consider: muscle contusion, muscle strain, muscle rupture, ligament sprain; send patient to X-ray and/or MRI to determine the extent of the injury or to identify possible additional injuries".

When the predicate $W_{9,7}$ is true, token $\alpha_{1}$ enters place $l_{7}$ and there it obtains a characteristic:

"consider: muscle cramps or delayed onset muscle soreness".

When the predicate $W_{9,8}$ is true, token $\alpha_{1}$ enters place $l_{8}$ and there it obtains a characteristic:

$$
\text { "perform physical examination". }
$$


The transition $Z_{4}$ has the following form:

$$
Z_{4}=\left\langle\left\{l_{5}, l_{8}, l_{13}\right\},\left\{l_{10}, l_{11}, l_{12}, l_{13}\right\}, r_{4}\right\rangle
$$

where

$$
r_{4}=\begin{array}{c|cccc} 
& l_{10} & l_{11} & l_{12} & l_{13} \\
\hline l_{5} & \text { false } & \text { false } & \text { false } & \text { true } \\
l_{8} & \text { false } & \text { false } & \text { false } & \text { true } \\
l_{13} & W_{13,10} & W_{13,11} & W_{13,12} & \text { true }
\end{array}
$$

and

- $W_{13,10}=$ "there is muscle weakness without loss of muscle mass";

- $W_{13,11}=$ "there is muscle weakness and loss of muscle mass";

- $W_{13,12}=\neg W_{13,10}$.

The tokens from all input places of transition $Z_{4}$ enter place $l_{13}$ and unite with token $\nu$ with the characteristic as mentioned above. On the next timemoment, token $\nu$ splits to two tokens - the same token $\nu$ that stays permanently in the place $l_{12}$, and token $\alpha_{1}$. When the predicate $W_{13,10}$ is true, token $\alpha_{1}$ enters place $l_{10}$ and there it obtains a characteristic:

"perform a laboratory tests to rule in potential pathologies associated with muscle pain and weakness".

When the predicate $W_{13,11}$ is true, token $\alpha_{1}$ enters place $l_{11}$ and there it obtains a characteristic:

"consider: muscular dystrophy; perform: muscle biopsy, aldolase test, creatine phosphokinase test".

When the predicate $W_{13,12}$ is true, token $\alpha_{1}$ enters place $l_{12}$ and there it obtains a characteristic:

"consider: fibromyalgia, psyhological disorders, spinal disorders, endocrine disorders, polymyalgia rheumatica".

The transition $Z_{5}$ has the following form:

$$
Z_{5}=\left\langle\left\{l_{10}\right\},\left\{l_{14}\right\}, r_{5}\right\rangle
$$

where

$$
r_{5}=\frac{l_{14}}{l_{10} \mid t r u e}
$$

The token from place $l_{10}$ of transition $Z_{5}$ enter place $l_{13}$ and there it obtains a characteristic:

"perform: erythrocyte sedimentation rate (ESR) test, serum alkaline phosphatase (SAP) test, creatine phosphokinase (CPT) test". 
The transition $Z_{6}$ has the following form:

$$
Z_{6}=\left\langle\left\{l_{14}, l_{19}\right\},\left\{l_{15}, l_{16}, l_{17}, l_{18}, l_{19}\right\}, r_{6}\right\rangle
$$

where

$$
r_{6}=\begin{array}{c|ccccc} 
& l_{15} & l_{16} & l_{17} & l_{18} & l_{19} \\
\hline l_{14} & \text { false } & \text { false } & \text { false } & \text { fals } & \text { true } \\
l_{19} & W_{19,15} & W_{19,16} & W_{19,17} & W_{19,18} & \text { true }
\end{array}
$$

and

- $W_{19,15}=$ "erythrocyte sedimentation rate (ESR) is high";

- $W_{19,16}=$ "serum alkaline phosphatase (SAP) is high";

- $W_{19,17}=$ "creatine phosphokinase (CPT) is high";

- $W_{19,18}=\neg W_{19,15} \vee \neg W_{19,16}$.

The tokens from all input places of transition $Z_{6}$ enter place $l_{19}$ and unite with token $\pi$ with the characteristic as mentioned above. On the next time-moment, token $\pi$ splits to two tokens - the same token $\pi$ that stays permanently in the place $l_{19}$, and token $\alpha_{1}$. When the predicate $W_{19,15}$ is true, token $\alpha_{1}$ enters place $l_{15}$ and there it obtains a characteristic:

"consider: polymyalgia rheumatica, systemic lupus erythematosus, myositis or secondary carcinomatosis".

When the predicate $W_{19,16}$ is true, token $\alpha_{2}$ enters place $l_{16}$ and there it obtains a characteristic:

"consider: liver diseases, osteoporosis, osteomalacia, viral infections, Pagets disease and hyperparathyroidism".

When the predicate $W_{19,17}$ is true, token $\alpha_{3}$ enters place $l_{17}$ and there it obtains a characteristic:

"consider: drug-induced disorders, inflammatory myopathies".

When the predicate $W_{19,18}$ is true, token $\alpha_{4}$ enters place $l_{18}$ and there it obtains a characteristic:

"consider: hypothyroidism, fibromyalgia, psyhogenic causes, myofascial pain syndrome".

The transition $Z_{7}$ has the following form:

$$
Z_{7}=\left\langle\left\{l_{15}, l_{16}, l_{17}, l_{18}\right\},\left\{l_{20}\right\}, r_{7}\right\rangle
$$

where 


$$
r_{7}=\begin{array}{l|l} 
& l_{20} \\
\hline l_{15} & \text { true } \\
l_{16} & \text { true } \\
l_{17} & \text { true } \\
l_{18} & \text { true }
\end{array}
$$

The tokens from the input places of transition $Z_{7}$ enter place $l_{20}$ and unite in one token $\beta_{1}$ with some of the characteristics obtained from the previous timestep. The token $\beta_{1}$ returns to transition $Z_{1}$ and enters to place $l_{3}$ to extend the personal record of the current patient.

\section{Conclusions}

The so described GN-model may provide a framework that can be used by primary care practitioners to guide diagnostic processes for patients with muscle pain, enabling more accurate and efficient identification of conditions that may lead to pain in the muscles and would assist in optimizing patient outcomes and more effective treatment. The presented in this paper GN-model of diagnostic algorithm for patient with muscle pain is a part of a series of studies for diagnosing through GN-modeling assistance and can be improved in multiple ways to yield better results. This model significantly improves the accuracy of the primary diagnosis and the reliability of the proposed algorithm.

\section{Acknowledgements}

Work presented here is partially supported by the Grants: DFNP-142/2016 "Program for career development of young scientists, BAS" and DN02/10 "New Instruments for Knowledge Discovery from Data, and Their Modelling"

\section{References}

[1] Atanassov, K.,: Generalized Nets. World Scientific, Singapore (1991)

[2] Atanassov, K.,: On Generalized Nets Theory. Prof. M. Drinov Academic Publishing House, Sofia (2007)

[3] Ribagin, S., Atanassov, K., Shannon, A.: Generalized Net Model of Shoulder Pain Diagnosis. Issues in Intuitionistic Fuzzy Sets and Generalized Nets 11, 55-62 (2014)

[4] Ribagin, S., Roeva, O., Pencheva, T.: Generalized Net Model of Asymptomatic Osteoporosis Diagnosing. Proc. of 2016 IEEE 8th International Conference on Intelligent Systems (IS), Sofia, 604-608 (2016)

[5] Shannon, A., Sorsich, J., Atanassov, K., Nikolov, N., Georgiev, P.: Generalized Nets in General and Internal Medicine, Vol. 1, Prof. M. Drinov Academic Publishing House, Sofia (1998) 